\title{
Benefícios da utilização da babosa (Aloe vera) na fitoterapia
}

\author{
Benefits of using Aloe vera in herbal medicine \\ Beneficios del uso de Aloe vera en la medicina herbal
}

Recebido: 02/12/2021 | Revisado: 07/12/2021 | Aceito: 08/12/2021 | Publicado: 09/12/2021

\author{
Maria dos Remédios Brito Nascimento \\ ORCID: https://orcid.org/0000-0001-9223-4442 \\ Universidade Estadual do Piauí, Brasil \\ E-mail: remeddynhabritto62@gmail.com \\ Rothchild Sousa de Morais Carvalho Filho \\ ORCID: https://orcid.org/0000-0002-7067-6159 \\ Universidade Estadual do Piauí, Brasil \\ E-mail: rothchildquimicahsb@gmail.com \\ Rosa Virgínia Soares Mamede \\ ORCID: https://orcid.org/0000-0002-2497-0736 \\ Universidade Estadual do Piauí, Brasil \\ E-mail: rosavirginia@prp.uespi.br
}

\begin{abstract}
Resumo
A presente revisão sistemática se refere a utilização de plantas na medicina. Para a maioria da população é uma busca de alternativa de cura. Os artigos publicados, entre 2000 e 2014, em três bases de dados científicas: Google acadêmico, Scielo e Web of Science, foram submetidos à análise que foi realizada inicialmente a partir da leitura do título do artigo, observando quais mencionavam termos relacionados com a fitoterapia. Os artigos selecionados na primeira etapa foram analisados por meio da leitura do Abstract, sendo selecionados os que mencionavam algum tipo de tratamento fitoterápico com o uso da babosa, Aloe vera L. Destes 36 artigos, 8 em inglês e 28 em português. Atualmente o interesse pelo benefício das plantas vem crescendo na área de homeopatia e na fitoterapia. Entre as mais utilizadas está a babosa (Aloe vera). Presente tanto na medicina popular como em cosméticos.
\end{abstract}

Palavras-chave: Fitoterapia; Plantas medicinais; Babosa (Aloe vera).

\begin{abstract}
This systematic review refers to the use of plants in medicine. For most of the population it is a search for an alternative cure. Articles published between 2000 and 2014 in three scientific databases: Academic Google, Scielo and Web of Science were subjected to analysis that was initially carried out by reading the title of the article, noting which mentioned terms related to herbal medicine. The articles selected in the first stage were analyzed by reading the Abstract, being selected those that mentioned some type of herbal treatment with the use of aloe vera L. Of these 36 articles, 8 in English and 28 in Portuguese. Currently, interest in the benefits of plants is growing in the field of homeopathy and herbal medicine. Among the most used is the aloe vera (Aloe vera). Present in both folk medicine and cosmetics.
\end{abstract}

Keywords: Phytotherapy; Medicinal plants; Aloe vera (Aloe vera).

\section{Resumen}

Esta revisión sistemática se refiere al uso de plantas en medicina. Para la mayoría de la población se trata de la búsqueda de una cura alternativa. Los artículos publicados entre 2000 y 2014 en tres bases de datos científicas: Google Académico, Scielo y Web of Science fueron sometidos a un análisis que inicialmente se llevó a cabo mediante la lectura del título del artículo, señalando que mencionaba términos relacionados con la fitoterapia. Los artículos seleccionados en la primera etapa fueron analizados mediante la lectura del Resumen, seleccionándose aquellos que mencionaban algún tipo de tratamiento a base de hierbas con el uso de aloe vera L. De estos 36 artículos, 8 en inglés y 28 en portugués. Actualmente, el interés por los beneficios de las plantas está creciendo en el campo de la homeopatía y la medicina herbal. Entre los más utilizados se encuentra el aloe vera (Aloe vera). Presente tanto en medicina popular como en cosmética.

Palabras clave: Fitoterapia; Plantas medicinales; Aloe vera (Aloe vera). 


\section{Introdução}

O uso de plantas medicinais pode ser entendido como uma prática que atravessa milênios, estando historicamente presente na sabedoria do senso comum, articulando cultura e saúde, uma vez que estes aspectos não ocorrem isoladamente, mas inseridos em um contexto histórico (Maharjan \& Nampoothir, 2014).

Valores naturais e ecológicos retornam com a determinação de novos preceitos nas áreas de conhecimento científico e da vida prática. Assim, o uso de plantas para fins medicinais tem sido renovado, provocando interesse pelos conhecimentos dos fármacos delas originados, incluindo a sua morfologia e composição química. Para que as plantas sejam utilizadas para fins terapêuticos, devem-se atender todos os critérios de eficácia, de segurança e qualidade além de apresentarem propriedades terapêuticas reprodutíveis e uma padronização em sua composição química (Silva et al, 2014).

As plantas medicinais tem sido utilizadas para tratamentos de enfermidades devido ao seu uso na medicina popular e pelas atividades farmacológicas. Assim, o homem tem se utilizado desse recurso para o tratamento de doenças, na forma de chás e medicamentos de manipulação (Caroline \& Luci, 2014).

Desde a antiguidade, os índios já preparavam seus medicamentos com plantas retiradas das florestas, da mesma forma, os benzedores, curandeiros e os xamãs, com o conhecimento herdado dos magos e feiticeiros do passado. O homem vem utilizando plantas para chegar à cura de várias doenças. Apesar das plantas medicinais fazerem parte da cultura popular, nas últimas décadas o interesse pela Fitoterapia teve um aumento considerável entre usuários e pesquisadores (Barbosa \& Pinto, 2003).

A Babosa também conhecida cientificamente como (Aloe vera). originária do hebraico, halal ou do arábico, alloeh (= substância amarga, brilhante) e do latim vera (= verdadeira). Esta planta é reconhecida como medicinal desde os tempos antigos (Ceolin et al, 2008).

Os antigos muçulmanos e judeus acreditavam que a babosa representava uma proteção para todos os males e, por isso, usavam as folhas penduradas nas portas na entrada de suas casas. Alexandre "O Grande", teria conquistado as ilhas de Socotorá, no Oceano Índico, aproximadamente no século IV. a. C, por possuir uma espécie de babosa que produzia uma tinta violácea. Há quem diga, que na verdade, o conquistador conhecia os poderes cicatrizantes da Babosa e o seu principal interesse nas ilhas era ter acesso às plantas para curar os ferimentos dos soldados após as batalhas (Palharrin et al, 2008).

Aloe barbadenses Miller (Aloe vera) é considerado como sendo a mais biologicamente ativa das espécies identificadas, cerca de 420, caracterizadas até esta data. Aloe vera contém vários constituintes ativos, incluindo vitaminas, minerais, polissacarídeos, amino ácidos, antraquinonas, saponinas, fitoesteróis, ácido salić́lico, etc (Yunes et al, 2015).

As babosas são de fácil cultivo, pois não são exigentes quanto ao solo, desde que este seja drenado e permeável (arenoso e areno-argiloso), mas são sensíveis à acidez. Conhecida há pelo menos três mil anos, somente no último século é que a misteriosa e mágica babosa conquistou o interesse da ciência oficial. Hoje em dia, vários centros de pesquisa nos hospitais e na indústria cosmética estão trabalhando para conhecê-la e aplicá-la nas suas múltiplas funções, como na fitoterapia, que é uma terapia exclusiva com plantas medicinais (Barbosa \& Pinto, 2003).

A Fitoterapia é a utilização de vegetais em preparações farmacêuticas. A fitoterapia vem do grego "tratamento" (therapeia) "vegetal" (phyton). É um tratamento exclusivo com uso de plantas medicinais em doenças, manutenção e recuperação da saúde (Juliana, 2015). A busca por terapias complementares é uma prática comum no Brasil, sendo um exemplo disso a utilização de plantas medicinais - fitoterapia. Pode-se definir como plantas medicinais aquelas que, administradas ao homem ou animais por qualquer via ou sobre qualquer forma, exercem alguma espécie de ação farmacológica. O tratamento fitoterápico, como qualquer outro, requer um diagnóstico correto do problema a tratar, para que a planta medicinal utilizada ofereça um resultados eficaz (Ceolin et al, 2008). Associadas às suas atividades terapêuticas estão seu baixo custo, a grande disponibilidade da matéria prima e a cultura relacionada ao seu uso. A prescrição de fitoterápicos até 
há pouco tempo não era aceita pelos próprios cientistas, por ser considerada uma medicina inferior. Ou seja, um tratamento barato, pois eles acreditavam que não havia benefício por causa do seu custo (Shaik et al, 2015). A atividade cicatrizante da Aloe vera é explicada por várias teorias. Estima-se que ela possua cerca de duzentas moléculas biologicamente ativas que atuam sinergicamente sobre os fibroblastos durante a formação de um novo epitélio. Sua ação cicatrizante é explicada pela presença do tanino que favorece a granulação e contração da ferida com mais eficiência (Ceolin et al, 2008).

Diante da importância da fitoterapia e da escassa difusão de pesquisa na Medicina, tornou-se necessário o estudo dos efeitos terapêuticos da Babosa (Aloe vera) popularmente utilizado pela sua ação cicatrizante. Depois de quase meio século de predomínio da medicina alopática e dos remédios sintéticos, as pessoas voltam sua atenção para os medicamentos naturais. Isto é uma nova consciência ecológica que surge, buscando o equilíbrio do homem com o meio ambiente, diminuindo os efeitos tóxicos dos remédios, substituindo-os pela fitoterapia; garantida e testada pelos chás, através dos tempos (Barbosa \& Pinto, 2003). O crescimento do mercado mundial de fitoterápicos é estimado de 10 a $20 \%$ ao ano e as principais razões que impulsionaram este aumento nas últimas décadas foram: valorização de uma vida de hábitos saudáveis, e, consequentemente, o consumo de produtos naturais. Os evidentes efeitos colaterais dos medicamentos sintéticos, a descoberta de novos princípios ativos nas plantas, a comprovação científica da fitoterapia e o preço de maneira geral ser mais acessível à população com menor poder aquisitivo favorecem o uso de plantas medicinais (Botelho et al., 2014). Neste sentido, é importante lembrar que o Ministério da Saúde Brasileiro, nos últimos anos, busca estimular a inclusão de práticas complementares saudáveis no sistema oficial de saúde. Destaca-se a implementação da Política Nacional de Plantas Medicinais e Fitoterápicas (PNPMF) e a Política Nacional de Práticas Integrativas e Complementares (PNPIC), e que visam estimular o acesso às práticas complementares e às plantas medicinais, para o cuidado da saúde, de forma eficaz e segura (Nafiseh \& Hossein, 2014).

O objetivo desse trabalho foi analisar as propriedades fitoterápica da babosa (Aloe vera). Enumera as atividades biológicas, ter acesso da importância dessa planta medicinal na elaboração de produtos terapêuticos para cicatrização de lesões e observar as frequências dos dados publicados cientificamente.

\section{Metodologia}

O presente estudo trata-se de uma análise de dados secundários que consistiram em artigos publicados em periódicos nacionais e internacionais entre 2000 a 2014 e pesquisadas nas bases de dado no Google acadêmico, Scielo, Web of sciencie e outros. A inclusão das publicações sobre plantas medicinais aplicadas em fitoterapia foi definida a partir da análise dos títulos, resumos e palavras-chave dos que continham o tema "Benefício da Babosa na fitoterapia", nos artigos publicados em periódicos nacionais e internacionais. Para a análise, foram utilizados artigos e mamografias. Este estudo pode ser caracterizado como exploratório, com método quantitativo. A pesquisa é do tipo documental, visto que o material objeto de estudo são artigos publicados em revistas científicas, um tipo de publicação periódica (Piriz et al, 2014).

Quanto aos métodos de pesquisa utilizados nos estudos publicados nos artigos analisados, os do tipo misto são a preferência da maioria dos autores. Este fato pode ser explicado, pois na maioria dos artigos o intuito dos autores era obter dados sobre os compostos presentes em plantas medicinais e a percepção sobre o consumo dessas plantas.

Após esta abordagem, os artigos foram separados para análise. Nessas condições, encontrou-se um total de 36 artigos científicos que passaram a integrar a segunda etapa da pesquisa, descritiva quantitativa. Nessa etapa foi identificada a planta medicinal, que no caso da revisão foi a Babosa (Aloe vera).

O método é revisar a utilidade das plantas em tratamentos fitoterápicos e a pesquisa utiliza os instrumentos de coleta de dados sobre a Babosa (Aloe vera), pois a mesma já vem deste os primórdios conhecidos como planta medicinal. 
Assim, procedeu-se à filtragem, por busca de artigos sobre o tema em questão, utilizando os seguintes critérios: periódicos avaliados por pares, artigos que apresentavam a expressão "Benefício da Babosa na fitoterapia" no título. Este estudo pode ser caracterizado como exploratório, com método quantitativo. Conforme o fluxograma a seguir: Figura 1.

Figura 1: Bases de dados.

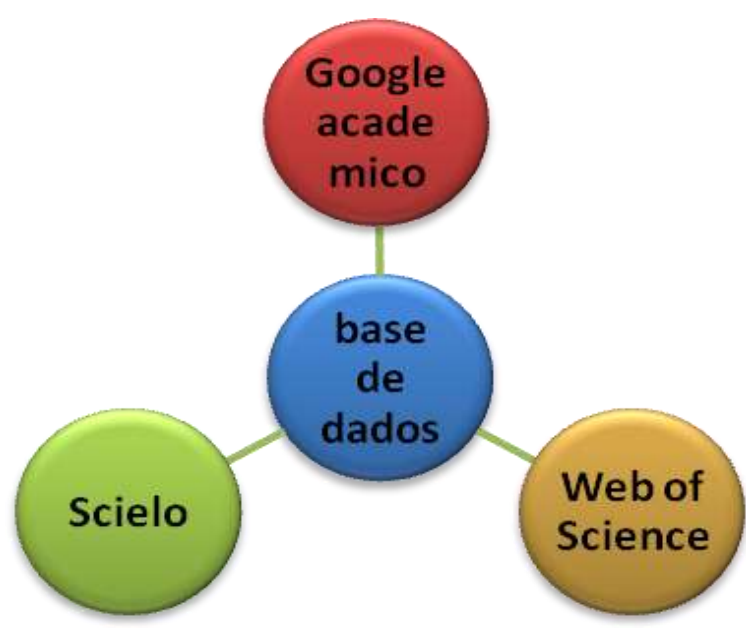

Fonte: Autores (2015).

\section{Resultados e Discussão}

Nos artigos analisados, não foi constatada a presença de um mesmo autor em mais de um artigo. Isso pode inferir a falta de referências no meio acadêmico quando o assunto tratado é sobre o beneficio da Babosa na fitoterapia, assim como pode estar diretamente ligado ao fato de a pesquisa sobre esses tratamentos fitoterápicos com o uso da mesma ser ainda recente no país. Nos artigos estudados, foi observado que em alguns obtiveram resultados bem mais precisos que em outros.

Quando questionados em relação ao tempo de tratamento fitoterápico 71,9\% afirmam que utilizam por um $\operatorname{logo}$ período de 86 dias aproximados, e 3,1\% afirma fazer uso por um curto período de 23 dias, sendo importante este dado, pois o uso de algumas plantas pode causar efeitos colaterais devido ao acúmulo de substâncias tóxicas num longo tempo de utilização. Assim, foi possível apresentar um conhecimento razoável de plantas medicinais como a Babosa (Aloe vera) em tratamentos fitoterápicos.

Como principais atividades farmacológicas, foram relatadas as atividades cicatrizante e anti-inflamatória, sendo possível a utilização da mesma para o tratamento de lesões. Em estudo experimental, em 2012, a Babosa (Aloe vera) foi a mais pesquisada nos últimos 14 anos, dentre os estudos clínicos e experimentais com plantas medicinais na cicatrização (Shaik et al, 2014).

Em análise de 2010 e 2014 foi apresentado um gel da babosa (Aloe vera) produzida para realizar teste, ficou à disposição para serem utilizados em lesões, em animais atendidos em Hospital Veterinário, dentre elas as mais frequentes eram as lesões provocadas por traumas e lesões pós-cirúrgicas, nos traumas, o curativo feito com o gel da babosa (Aloe vera), usando gazes e atadura, sendo realizadas duas trocas duas vezes ao dia até a cicatrização durantes 7 dias. Em cirurgias realizadas nesses animais com gel (Aloe vera) diretamente no ferimento cirúrgico e recoberto com gases micro porosa uma única vez, até a retirada dos pontos que o curativo era retirado após 7 dias (Paul \& London, 2016). Na avaliação final observou-se que não houve deficiência, secreção e nem edema nos tecidos cicatrizados. Apenas um curativo com o gel da babosa foi suficiente para uma total cicatrização das feridas pós-cirúrgicas provocadas (Mushafau et al, 2015). 
Conforme foi descrito nos trabalhos de monografia de Juliana M, M em 2010 e no artigo de Mayra, K, M de S; et al, em 2013 observou-se que a produção do gel de babosa (Aloe vera), comparada a uma solução fisiológica, os resultados foram diferentes. Observe a Figura 2 monstra dois tipos de tratamento (Piriz et al, 2014).

Figura 2: Gráfico mostrando as médias das áreas de retração das feridas tratadas com gel da babosa (GT) e solução fisiológica (GC).

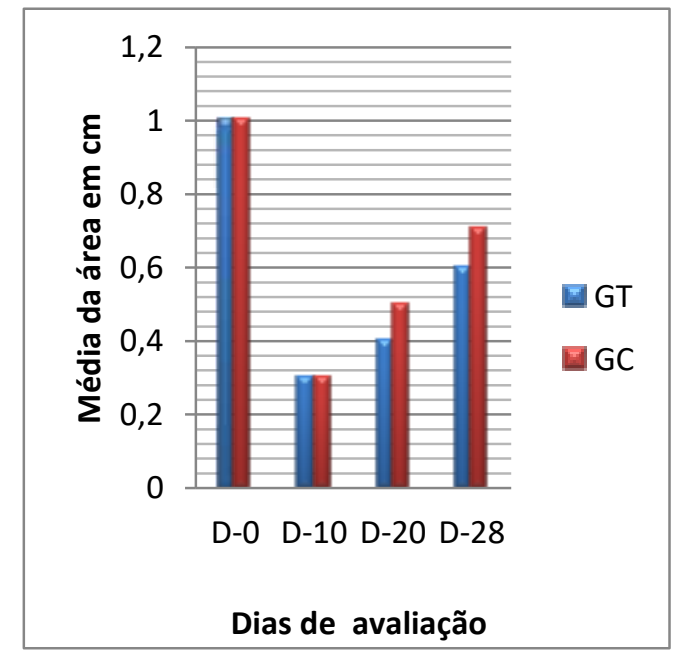

Fonte: Autores (2015).

A partir dos dados pesquisados de trabalhos monográficos e artigos, a pesquisa teve as seguintes decorrências: a lesão adquirida por traumas e lesões adquiridas através de cirurgias teve tratamentos diferenciados (8). Entretanto, a babosa teve um resultado com eficiente semelhante ao da solução fisiológica. A GT é o grupo de tratamentos com o uso do gel produzido pela babosa (Aloe vera), onde a eficiência de cicatrização foi maior do que a de GC, que é o grupo de controle em que o tratamento é realizado com um soro fisiológico (Souza et al, 2014).

Entre D-0 e D-10, demonstraram as mesmas atividades significativas em ambos. Entre D-10 e D-20, as atividades apresentaram bons desempenhos, tanto no soro como em babosa. Entre D-20 e D-28 não houve diferença perceptível entre os tratamentos (Piriz et al, 2014).

A Tabela 1 mostra os resultados encontrados em cada uma das bases de busca da pesquisa. Dessa forma, foram selecionados 75 artigos de estudos científicos com plantas medicinais na cicatrização, em consideração a babosa (Aloe vera) em tratamentos fisioterápicos. Para apresentação no presente trabalho, foram excluídos 43 artigos, e acrescentou-se 7 artigos, devido à sua relevância científica e benefícios para o conhecimento do potencial da babosa (Aloe vera), totalizando 36 artigos (Parente et al, 2008). 
Tabela 1: Artigos científicos. Nacionais e Internacionais, período de 2000-2014.

\begin{tabular}{lrccc}
\hline $\begin{array}{c}\text { Base de } \\
\text { dados }\end{array}$ & $\begin{array}{r}\text { Total de } \\
\text { artigos }\end{array}$ & $\begin{array}{r}\text { Excluídos após leituras } \\
\text { de títulos e resumos }\end{array}$ & $\begin{array}{c}\text { Lidos na } \\
\text { íntegra }\end{array}$ & Selecionados \\
\hline Google acadêmico & 31 & 21 & 18 & 13 \\
Scielo & 18 & 10 & 12 & 11 \\
Web of Science & 15 & 07 & 11 & 09 \\
Outros & 11 & 05 & 06 & 03 \\
TOTAL & 75 & 43 & 47 & 36 \\
\hline
\end{tabular}

Fonte: Autores (2015).

Tabela 2: Relação dos artigos da revisão de acordo com autores, ano de publicação, título do periódico e país.

\begin{tabular}{|c|c|c|c|c|}
\hline \multicolumn{5}{|c|}{ Nomes Tabelados. } \\
\hline & Ano & Autores & Titulo & Pais \\
\hline 01 & 2003 & $\begin{array}{l}\text { Barbosa }^{\mathrm{a}}, \quad \text { W.L.R.1*; } \\
\text { Pinto, L.N.2. }\end{array}$ & $\begin{array}{l}\text { Documentação e variação da fitoterapia tradisna Kaiap nas aldeias A“Ukre } \\
\text { e pycanu - sudeste do Pará. }\end{array}$ & Brasil \\
\hline 02 & 2006 & $\begin{array}{l}\text { Caroline F; Eliane, B. F; } \\
\text { Luci, F. R. }\end{array}$ & $\begin{array}{l}\text { Uso de plantas medicinais como alternativa fitoterápica nas unidades de } \\
\text { saúde pública de Santa Teresa e Marilândia, ES3 }\end{array}$ & Brasil \\
\hline 03 & 2008 & $\begin{array}{l}\text { Ceolin,T; Zillmer, J, G, } \\
\text { V; Arrieira, I, C; } \\
\text { Heck,R,M; Barbieri, R, } \\
\text { L. }\end{array}$ & $\begin{array}{l}\text { Utilização de babosa (ALOE spp) no cotidiano de usuários prestadores de } \\
\text { câncer. }\end{array}$ & Brasil \\
\hline 04 & 2008 & $\begin{array}{l}\text { Palharin, L, H, Di, C } \\
\text { Figueiredo Neto, E } 1 \text {; } \\
\text { Camargo Lopes, M, P } \\
\text { Ascencio. }\end{array}$ & Efeitos fitoterápicos e hepáticos de babosa. & Brasil \\
\hline 05 & 2009 & $\begin{array}{l}\text { Brandão MGL1,2 et al., } \\
2009 .\end{array}$ & $\begin{array}{l}\text { Biodiversidade, Uso Tradicional de Plantas Medicinais e Produção de } \\
\text { Fisioterápicos em Minas Gerais }\end{array}$ & Brasil \\
\hline 06 & 2010 & Juliana, M, M. & $\begin{array}{l}\text { Monografia submetida ao Curso de Medicina Veterinária como requisito } \\
\text { parcial para obtenção do grau de Médica Veterinária }\end{array}$ & Brasil \\
\hline 07 & 2011 & Argenta et al.,2011. & Plantas Medicinais: Cultura Popular Versus Ciência & Brasil \\
\hline 08 & 2006 & $\begin{array}{l}\text { Alvim, N.A.T. et al., } \\
\text { 2006. }\end{array}$ & $\begin{array}{l}\text { O uso de plantas medicinais como recurso terapêutico: das influências da } \\
\text { formação profissional às implicações éticas e legais de sua aplicabilidade } \\
\text { como extensão da prática de cuidar realizada pela enfermeira. }\end{array}$ & $\begin{array}{l}\text { Latino- } \\
\text { Americana }\end{array}$ \\
\hline 09 & 2012 & $\begin{array}{l}\text { Sonia, M. J. Ortiz, } \\
\text { Dra }^{\text {a }} \quad \text { Alcimara A. } \\
\text { Foetsch. }\end{array}$ & $\begin{array}{l}\text { Saber ambiental: Influências das plantas medicinais e dos Fitoterápicos } \\
\text { através da atuação de benzedeiras e Pastorais da Saúde na região do } \\
\text { Contestado }\end{array}$ & Brasil \\
\hline 10 & 2013 & $\begin{array}{l}\text { Mayra, K, M de S; et } \\
\text { al,.2013. }\end{array}$ & $\begin{array}{l}\text { Produção do gel da babosa (Aloe vera) para cicatrização de feridas } \\
\text { cutâneas de cães e gatos. }\end{array}$ & Brasil \\
\hline 11 & 2014 & $\begin{array}{l}\text { Botelho }^{2}, \mathrm{~N}, \mathrm{M} ; \text { Brito }^{3}, \\
\text { N, B; Silva }\end{array}$ & $\begin{array}{l}\text { A utilização de plantas medicinais pela comunidade e comunidade de cana } \\
\text { de visconde. }\end{array}$ & Brasil \\
\hline 13 & 2014 & Nafish;et al,.2014. & Qualitative improvement of low meat beef burger using Aloe vera. & Iran. \\
\hline 14 & 2014 & $\begin{array}{lr}\text { Maharjan H. } & \text { Radha, } \\
\text { Nampoothiri } & \text { P. } \\
\text { Laxmipriya* } & \\
\end{array}$ & Evaluation of biological properties and clinical effectiveness of Aloe vera: & Índia \\
\hline 15 & 2014 & 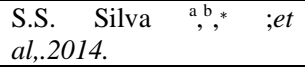 & Bio-inspired Aloe vera sponges for biomedical applications. & Portugal \\
\hline 16 & 2014 & 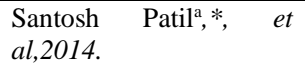 & $\begin{array}{l}\text { Comparative evaluation of natural antioxidants spirulina and Aloe vera for } \\
\text { the treatment of oral submucous fibrosis. }\end{array}$ & $\begin{array}{l}\text { Arábia } \\
\text { Saudita }\end{array}$ \\
\hline 17 & 2015 & $\begin{array}{l}\text { Shaik Abdul Hussain }{ }^{\mathrm{a}}, * \\
\text { et al, } 2015 .\end{array}$ & $\begin{array}{l}\text { Ingredient formulation effects on physico-chemical, sensory, textural } \\
\text { properties and probiotic count of Aloe vera probiotic dahi. }\end{array}$ & China \\
\hline 18 & 2015 & $\begin{array}{l}\text { Yunes Panahi, et al, } \\
2015 .\end{array}$ & $\begin{array}{l}\text { Efficacy and safety of Aloe vera syrup for the treatment of } \\
\text { gastroesophageal Reflux disease: a pilot randomized positive-controlled }\end{array}$ & Índia \\
\hline
\end{tabular}

Fonte: Autores (2015). 
Nesta pesquisa bibliográfica, os países que merecem destaque por pesquisarem sobre os efeitos de plantas medicinais, tendo a babosa (Aloe vera) inclusa em suas pesquisas e em tratamentos fitoterápicos, sendo os interesses em tratamento dinâmicos para que tenham resultados em cicatrização são: em Brasil, China, Irã, Arábia Saudita e Portugal. Dos 36 artigos listados na Tabela 2 são os que satisfazem. Pois a Tabela 2 está organizada da seguinte forma: ano que o artigo foi publicado, o nome do autor, o tema do artigo e o país onde a pesquisa foi realizada.

Segundo estudo de Caroline et al. desenvolvido em sua literatura na cidade de João Pessoa (PB), doze pessoas portadoras de câncer faziam o uso da babosa por ter a possibilidade de cura. Já que nove pessoas que estavam nessa pesquisa sabiam sobre a possibilidade de utilização da babosa para o câncer, e apenas três referiram terem utilizado para auxiliar no tratamento (Caroline \& Luci, 2014).

A através de hábitos vindo dos antepassados e de diversas culturas que vem atravessando o tempo, em uma pesquisa realizada em 2003 por (Barbosa \& Pinto, 2003), trabalho realizado no Brasil que apresenta o tema: Documentação e variação da fitoterapia tradisna Kaiap nas aldeias A“Ukre e pycanu - sudeste do Pará. Sendo um trabalho realizado para conhecer o uso e preparo de medicamentos naturais pela comunidade assistida pela USF canal da Visconde, foi observado que $95 \%$ dessas pessoas faz uso de plantas medicinais, e entre essas plantas está a babosa (Aloe vera) como umas das mais usada.

O mesmo foi observado em diversos outros trabalhos citados na Tabela 2. Pesquisas desenvolvidas no Brasil e Londres e entre outros, identificaram a eficácia do uso da babosa (Aloe vera) em tratamentos fitoterapia em pessoas portadoras de células cancerosas e entre outros sintomas, sendo que $57 \%$ teve resultados benéfico, e os outros por não terem seguidos as normas do tratamento não tiveram nenhum beneficio. Os presentes estudos acompanham o estilo de vida, pois os mesmos que estão mais incluídos desses usos de plantas medicinais são pessoas de baixa rendas.

Com base no que foi exposto, as plantas medicinais tornam uma alternativa de grande relevância para o processo de cicatrização, que começam a chama á atenção da saúde não só dos Brasileiros e sim do mundo inteiro, considerando que seu uso seja válida para todos as pesquisas que demonstre seu potencial de cicatrizar, o que faz novas sugestões de comprovação, custos e benefícios, e a constate atualização das publicação realizadas.

\section{Conclusão}

Diante da ampla biodiversidade de plantas utilizadas em benefício da população, torna-se necessário uma melhor distribuição de conhecimento dos resultados provocados pelas mesmas, bem como a segurança no uso de plantas como recurso fitoterápico. Neste argumento, pode-se destacar que a pesquisa voltada para o campo das plantas medicinais é destaque a babosa (Aloe vera) na aplicação da fitoterapia, pois a utilização de plantas medicinais vem sendo acionada em programas de saúde. Um exemplo é o SUS (Sistema Único de Saúde). Dessa forma vai criando uma relação entre universidades e comunidades para que venham a formar um acrescentamento na qualidade de vida da população de baixa renda que dependem desses recursos terapêuticos.

\section{Referências}

Alenca, K. F. D. S., Soares, K. B. \& Alvarenga, E. M., Etnobotânica e educação não formal: usos e ensinamentos sobre plantas medicinais em Palmeirais-PI. Campinas, p. 07.

Barbosa, W. L. R. 1., Pinto, L.N. (2003). Documentação e variação da fitoterapia tradisna Kaiap nas aldeias A Ukre e pycanu - sudeste do Pará. Rev. Bras. Farmacogn., 13, 47-49.

Botelho, N. M., Brito, N. B. \& Silva, N. M. (2014). A utilização de plantas medicinais pela comunidade e comunidade de cana de visconde. Revista Paranaense de medicina. 28(1).

Caroline, F., Eliane, B. F., \& Luci, F. R., Uso de plantas medicinais como alternativa fitoterápica nas unidades de saúde pública de Santa Teresa e Marilândia, ES3. Natureza on line 4(1): 30-39. 10p. http://www.naturezaonline.com.br. 
Research, Society and Development, v. 10, n. 16, e470101624244, 2021

(CC BY 4.0) | ISSN 2525-3409 | DOI: http://dx.doi.org/10.33448/rsd-v10i16.24244

Ceolin, T., Zillmer, J. G. V., Arrieira, I. C., Heck, R. M., \& Barbieri, R. L. (2008). Utilização de babosa (ALOE spp) no cotidiano de usuários prestadores de câncer. 04p.

Heck, R. M., \& Barbieri, R. L., Utilização de babosa (ALOE spp) no cotidiano de usuários prestadores de câncer.

Juliana, M. M. Uso da babosa (Aloe vera) na reparação de feridas abertas provocadas cirurgicamente em cães, de campina grande centro de saúde e tecnologia rural campus de patos-PB curso de medicina veterinária.

Lorenzi, H, \& Matos, F. J. (2002). Plantas medicinais no Brasil: nativas e exóticas. Instituto Plantarum, 2002. 512p.

Maharjan, H. R., \& Nampoothir, P. L. (2014). Evaluation of biological properties and clinical effectiveness of Aloe vera: A systematic review.

Maria, G. D. Plantas medicinais, Fitoterápicos e saúde publica: um diagnostico situacional em Anápolis, Goiás.

Marmitt, D. J, Rempel, C, Goettert, M. I., \& Silva A. C. (2015). Revisão sistemática sobre a produção cientifica de plantas medicinais da Renisus voltadas ao diabetes mellitus. Caderno Pedagógico, 12(2), 87-99.

Mushafau, A. A. B, Joo, K. G. S. P. L., \& Adeline, Y. T. (2015). Metagenomics study of endophytic bacteria in Aloe vera using next-generation technology,

Nafiseh, S., \& Hossein, G. E. (2014). Qualitative improvement of low meat beef burger using Aloe vera.

Palharrin, L. H. C., Figueiredo, N. E., Camargo, L. M. P. \& Bosque, G. G. (2015). Efeitos fitoterápicos e hepáticos de babosa. (14).

Parente, L. M. L., Arneitro, L. M., Tresvenzol. \& Gardin, N. E. Aloe vera: botanical, phytochemical and therapeutic characteristics.

Paul, Caul. \& London U. K. (2016). Aloe vera, a natural cancer soother?

Piriz, M. A, Lima, C. A. B, Jardim, V. M, Mesquita, M. K., Souza, A. D. Z. \& Heck, R. M. (2014). Plantas medicinais no processo de cicatrização de feridas: uma revisão de literatura. Rev. Bras. Pl. Med., 16(3), 628-636.

Sousa, M. K. M., Cavalcante, S, P. C., Sousa, C. I., Silva. T. R., Amaral, C. R. A. \& Coelho, M. C. O. C., Produção do gel da babosa (Aloe vera) para cicatrização de feridas cutâneas de cães e gatos. XIII Jornada de ensino, pesquisa e extensão - Jepex 2013 - UFRPE: Recife, 09 a 13 de dezembro.

Santos, P. A., \& Bader, K. A-Z. (2015). Comparative evaluation of natural antioxidants spirulina and aloe vera for the treatment of oral submucous fibrosi,

Shaik, A. H. A., Girdhari, R. P. A, Ashidh, K. S. A. (2015). Ingredient formulation effects on physico-chemical, sensory, textural properties and probiotic count of Aloe vera probiotic dahi,

Silva. A. B. M. B. Oliveira. A. B., \& Reis A. B. (2015). Bio-inspired Aloe vera sponges for biomedical applications,

Souza, C. M. P, Brandão, D. O, Silva, M. S. P, Palmeira, A. C, Simões, M. O. S., \& Medeiros, A. C. D. Utilização de Plantas Medicinais com Atividade Antimicrobiana por Usuários do Serviço Público de Saúde em Campina Grande - Paraíba. Rev. Bras. Pl. Med., 15(2), 188-193.

Yunes, P., Hossein, K., Ghasem, V., Reza, M., \& Amirhosseien, S. (2015). Efficacy and safety of Aloe vera syrup for the treatment of gastroesophageal reflux disease: a pilot randomized positive-controlled trial. 35 . 